\title{
THEORETICAL PREDICTION OF MICROGRAVITY IGNITION DELAY OF POLYMERIC FUELS IN LOW VELOCITY FLOWS
}

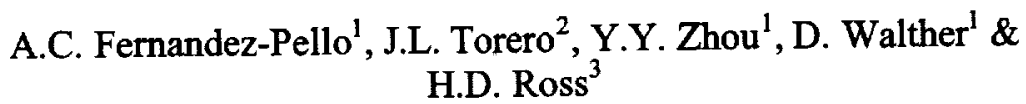 \\ ${ }^{1}$ University of California, Berkeley, CA 947201740 \\ ${ }^{2}$ University of Maryland, College Pak, MD 20742-3031 \\ ${ }^{3}$ NASA Glenn Research Center, Cleveland, OH 44135-3191
}

\section{Overview}

A new flammability apparatus and protocol, FIST (Forced Flow Ignition and Flame Spread Test), is under development. Based on the LIFT (Lateral Ignition and Flame Spread Test)[1] protocol, FIST better reflects the environments expected in spacebased facilities. The final objective of the FIST research is to provide NASA with a test methodology that complements the existing protocol [2] and provides a more comprehensive assessment of material flammability of practical materials for space applications. Theoretical modeling, an extensive normal gravity data bank and a few validation space experiments will support the testing methodology. The objective of the work presented here is to predict the ignition delay and critical heat flux for ignition of solid fuels in microgravity at airflow velocities below those induced in normal gravity. This is achieved through the application of a numerical model previously developed $\beta$ ] of piloted ignition of solid polymeric materials exposed to an external radiant heat flux. The model predictions will provide quantitative results about ignition of practical materials in the limiting conditions expected in space facilities.

Experimental data of surface temperature histories and ignition delay obtained in the $\mathrm{KC}-135$ aircraft $[4,5]$ are used to determine the critical pyrolysate mass flux for ignition and this value is subsequently used to predict the ignition delay and the critical heat flux for ignition of the material. Surface temperature and piloted ignition delay calculations for Polymethylmethacrylate (PMMA) and a Polypropylene/Fiberglass (PP/GL) composite were conducted under both reduced and normal gravity conditions. It was found that ignition delay times are significantly shorter at velocities below those induced by natural convection.

\section{Introduction}

Long-term missions in space facilities bring concern about the possibility of an accidental fire $[6,7]$, since there are combustible materials and sources of ignition aboard. In fact, there have been some minor incidents reported on Space Shuttle flights [8] and aboard MIR [9].

Space facilities typically have low air currents of the order of $0.1 \mathrm{~m} / \mathrm{s}$ induced by HVAC systems, and variable oxygen concentration due to $\mathrm{CO}_{2}$ scrubbers. Unfortunately, natural convection flows induced by gravity impose a limitation on the lowest velocities that can be tested on earth, and consequently on determining microgravity fire properties. Moreover, ground based microgravity facilities can only provide short periods of reduced gravity, e.g. about 25 seconds for a NASA KC-135

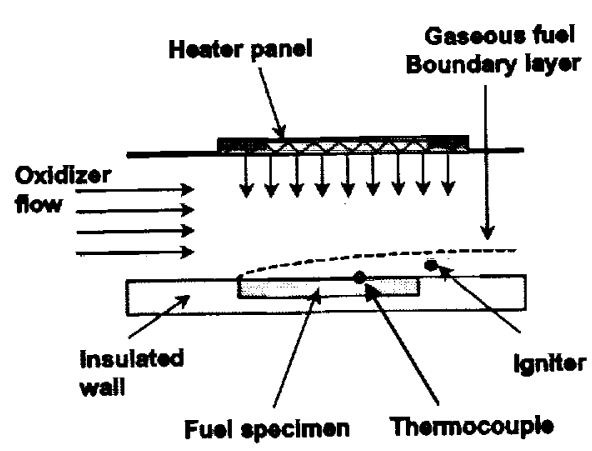

Figure 1. Schematic of the FIST configuration 
aircraft. Therefore, ignition tests in these ground based facilities can only be conducted at very high heat fluxes, which result in ignition ddays less than this time. Thus, until experiments are conducted in space based facilities, the results must be extrapolated, and are consequently limited in scope. Theoretical modeling, therefore, becomes a promising means to predict fire properties under microgravity and low velocity conditions.

Ignition of solid combustible materials has been extensively investigated theoretically [10-13]. Many investigators have focused on the condensed phase by introducing an empirical criterion for ignition. Among these, the critical pyrolysate mass flow rate at ignition is physically the most relevant $[10,14-16]$, because it is logically related to the lean flammability limit. For this reason, this criterion is adopted here in conjunctionwith the available experimental data. This strategy assumes fast chemistry in the gas phase.

\section{Problem configuration}

A schematic of the FIST apparatus is shown in Fig. 1. It consists of a small-scale combustion wind tunnel with a fuel sample mounted into a low conductivity, inert slab on one wall of the test section, and a radiant panel on the opposite wall. The surface of the fuel sample is impulsively exposed to a uniform and constant, external radiant heat flux, $5-40 \mathrm{~kW} / \mathrm{m}^{2}$. A known oxidizer forced flow is established parallel to the sample surface. A pilot is located downstream of the fuel sample. More detail is given in [4]. Experiments are conducted with two different fuels in both normal and reduced gravity. The first is

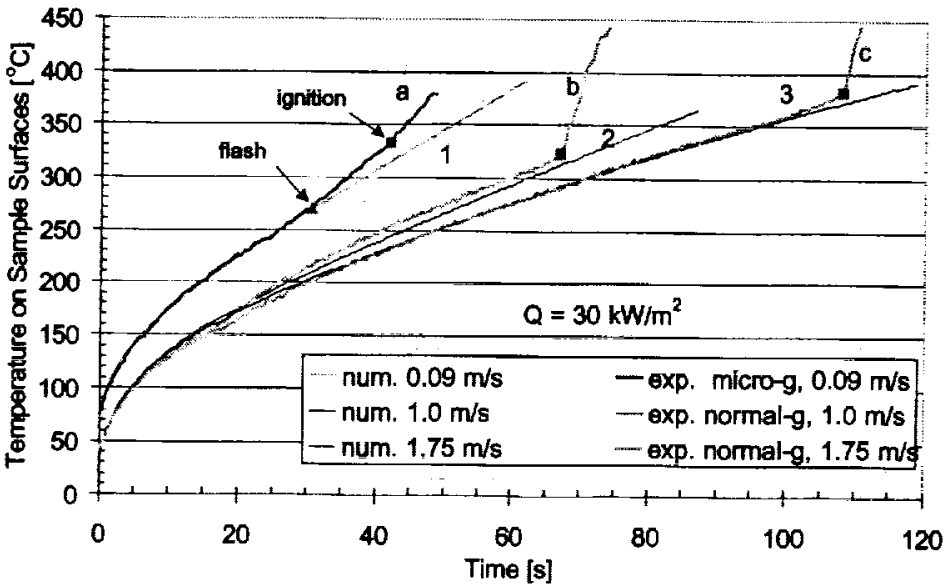

Figure 2. PP/GL surface temperatures in normal and reduced gravity at various airflow velocities.

\section{clear, 1/2" thick PMMA}

and the other is a $1 / 8$ "thick commercially made polypropylene ( $70 \%$ by mass) and loose glass fiber $(30 \%)(\mathrm{PP} / \mathrm{GL})$ blend, typically used for electronic casings and paneling.

Normal gravity tests are conducted at airflow velocities of $1.0,1.75$ and $2.5 \mathrm{~m} / \mathrm{s}$; larger than the limited values of natural convection. The experiments at forced airflow of 0.09 and $0.15 \mathrm{~m} / \mathrm{s}$ are conducted in a KC-135 aircraft following parabolic flight trajectories. The FIST testing apparatus is similar to the normal gavity test facility, albeit slightly reduced in scale [17] and is placed within the Spacecraft Fire Safety Facility (SFSF)[18]. Although these experiments are subject to the g-jitter and resulting buoyant flows of the aircraft, zero gravity and no buoyancy is assumed in modeling the low gravity experiments. The governing equations, boundary conditions, and the properties of PMMA and PP/GL can be found in [3] and [5]. Both thermal and oxidative pyrolysis are considered in the model together with in-depth absorption and phase change when appropriate.

For ignition to occur, the solid must first pyrolyze. The pyrolized fuel then mixes with the oxidizer in the boundary layer to produce a flammable mixture, which is ignited 
by the pilot. In order to determine the pyrolyzate mass flow rate at which ignition occurs, the numerical and FIST experimental surface temperature are compared at each specific airflow velocity. The value of the pyrolysate mass flow rate at the experimentally observed ignition time is considered to be the critical pyrolysate mass flow rate for

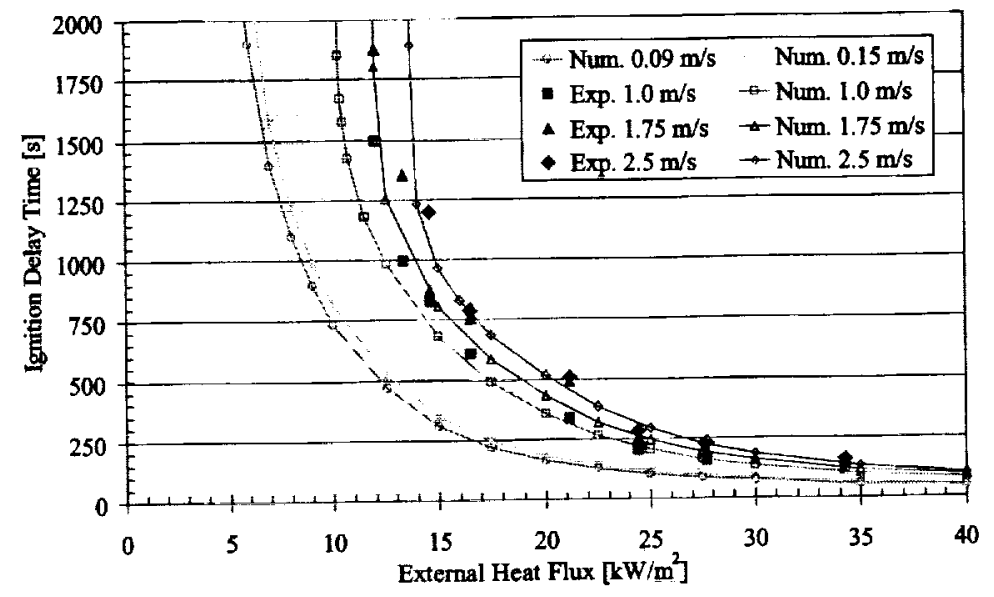

Figure 3. Experimental (solid) and predicted (open) ignition delay data for PMMA. those particular test conditions. This value is subsequently used to predict the ignition delay diagram (ignition delay vs. heat flux) and the critical heat flux for ignition.

\section{Results and discussion}

The measured and predicted surface temperature histories at various airflow velocities are shown in Fig. 2 for PP/GL composite $\left(30 \mathrm{~kW} / \mathrm{m}^{2}\right)$. The numerical results agree well with the experimental data up to the point of ignition. The experimentally observed sharp increase in temperature at ignition is not predicted by the model since the gas phase is not considered. Similar temperature histories are observed for PMMA[5].

The numerically predicted and experimentally measured ignition delays are plotted in Fig. 3 for PMMA and Fig. 4 for PP/GL. The critical heat fluxes can be deduced from the ignition diagrams by noting the external radiant flux at which the ignition delay approaches infinity. The ignition delay and critical heat flux for ignition decrease as the forced-flow velocity decreases, consistent with the findings of [16]. This suggests that

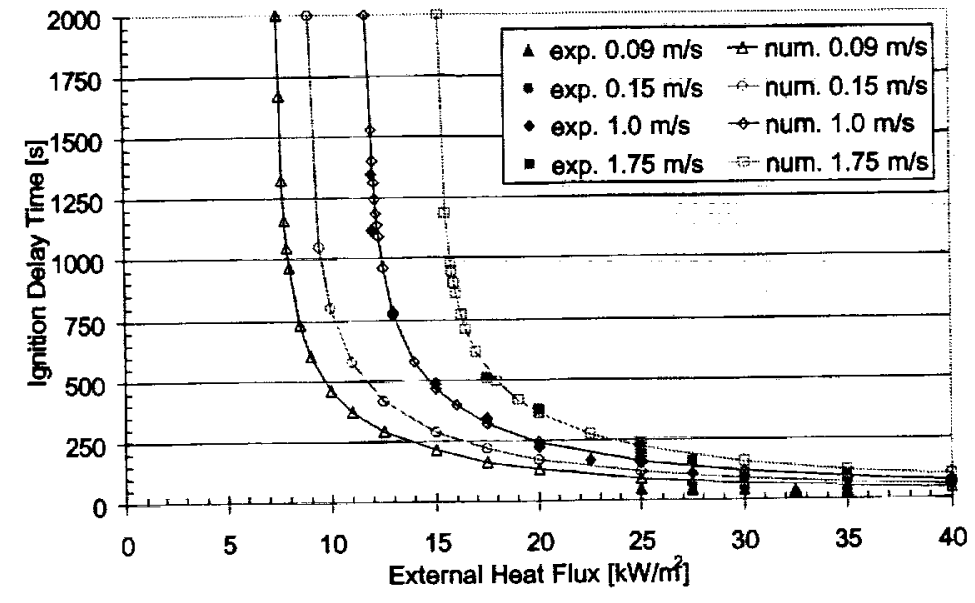

Figure 4. Experimental (solid) and predicted (open) ignition delay data for PP/GL. materials will ignite more easily under the low velocity conditions of space facilities. The critical heat flux for ignition as the forced flow velocities approach zero is close to half of the value measured in normal gravity. It should also be noted that since the gas phase is not considered in this model, oxygen limitation effects are not examined. 


\section{Conclusions}

The ignition delay, critical pyrolysate mass flow rate, and critical heat flux for ignition depend strongly on the airflow, and decrease as the airflow velocity decreases. This indicates that the environmental conditions must be considered in determining their values. In microgravity conditions, where airflows can be reduced significantly below natural convection limited values, the ignition delay times and the critical heat fluxes have been predicted to be close to half of the value in normal gravity. The results, if confirmed with longer duration microgravity tests, have very important implications since they indicate that materials will ignite easier under the conditions expected in space facilities, and that consequently stricter design specifications may be needed for fire safety.

\section{Acknowledgments}

This work was supported by NASA under Grant NCC3-478. The authors thank Messrs. S. Olenick, M. Roslon, J. Beck, A. Stevanovic, and T. Steinhaus for their experimental work. The authors would also like to thank the NASA reduced gravity aircraft team and support staff.

\section{References}

1. Annual Book of ASTM Standards, Vol. 04-07, 1055-1077 (1993).

2. Friedman, R., NASA TM-1999-209285.

3. Zhou, Y., and Fernandez-Pello, A. C., $29^{\text {th }}$ Symp. (Int.) on Combust., The Combust. Institute (2000)

4. Roslon, M., Olenick, S., Walther, D., Torero, J.L., Fernandez-Pello, A.C., and Ross, H.D., $38^{\text {th }}$ ALAA Space Sciences \& Exhibit, AIAA 2000-0580 (2000).

5. C. Fernandez-Pello, Y. Zhou, D. Walther; J. Torero, and H. Ross, $39^{\text {th }}$ AIAA Space

Sciences \& Exhibit, AIAA 2001-0471 (2001).

6. Palmer, H., IntI Microgravity Combustion Workshop, NASA LeRC, Cleveland, OH (1987).

7. Faeth, G., Space Station Freedom Modular Combustion Facility Assessment

Workshop, NASA LeRC, Cleveland, OH (1989).

8. Friedman, R, NASA TM 106403 (1994).

9. Ross, H. D.: "Burning To Go: Combustion On Orbit and Mars", Fall Technical

Meeting of the Eastern States Section of The Combustion Institute, Hartford CT (1997).

10. Atreya, A., Phil. Trans. R. Soc. Lond. A 356:2787-2813 (1998).

11. Di Blasi, C., Crescitelli, S., Russo, G., and Cinque, G., Combust. Flame, 83:333-344 (1991).

12. Nakabe, K., McGrattan, K. B., Kashiwagi, T., Baum, H. R., Yamashita, H., and

Kushida, G., Combust. Flame, 98:361-374 (1994).

13. Park, S. H., and Tien, C. L., Combust. Sci. Tech. 95:173-192 (1994).

14. Bamford, C. H., Crank, J., and Malan, D. H., Proc. Camb. Phil. Soc. 42:166-182 (1946).

15. Drysdale, D. D., and Thomson, H. E., Fire Safety Journal, 14:179-188 (1989).

16. Cordova, J.L. and Fernandez-Pello, A.C., Combust. Sci. Tech. 156:271-289 (2000)

17. Long, R.T., Quintiere, J.G, Torero, J.L. and Fernandez-Pello, A.C., Fire Safety Sci.6th Intl Symp., pp.567 (2000).

18. Goldmeer, J.S., NASA, TM-2000-210375. 